\title{
Wall-bounded turbulent shear flow: Analytic result for a universal amplitude
}

\author{
Amit Kr. Chattopadhyay and Jayanta K. Bhattacharjee \\ Department of Theoretical Physics, Indian Association for the Cultivation of Science, Jadavpur, Calcutta 700 032, West Bengal, India
}

(Received 22 February 2000; revised manuscript received 26 May 2000; published 21 December 2000)

\begin{abstract}
In the turbulent boundary layer above a flat plate, the velocity profile is known to have the form $v$ $=v_{0}[(1 / \kappa) \ln z+$ const $]$. The distance from the wall in dimensionless units is $z$ and $v_{0}$ is a uniquely defined velocity scale. The number $\kappa$ is universal, and measurements over several decades have shown that it is nearly 0.42 . We use a randomly stirred model of turbulence to derive the above law and find $\kappa=\sqrt{108 / 125 \pi} \simeq 0.52$.

DOI: 10.1103/PhysRevE.63.016306

PACS number(s): 47.27.Nz, 64.60.Ht
\end{abstract}

\section{INTRODUCTION}

Turbulent flow over a stationary plate has been investigated in great detail for well over a century. One of the important quantities studied is the velocity profile, and in particular a good deal of attention has been paid to the velocity in the boundary layer. The existence of the boundary layer was first postulated by Prandtl [1,2]. Due to the presence of viscosity, the flow speed has to be zero on the surface of the plate. Within a short distance, the velocity has to rise to its bulk value. This small distance in which the velocity experiences a rapid change is called the boundary layer. Prandtl observed that in the boundary layer the velocity $v$ scales with the distance $z$ from the boundary. Adjoining the boundary is an extremely thin laminar boundary layer, after which comes a buffer zone and then the turbulent boundary layer. In this regime one has $[2,3]$

$$
\frac{v}{V_{0}}=\frac{1}{\kappa}\left[\ln \left(z / z_{0}\right)\right],
$$

a result known as the law of the wall, where $V_{0}$ is a reference velocity to be defined below, and $\kappa$ is an universal constant which so far has only been experimentally measured [4].

With the coming of Kolmogorov's theory [5] of homogeneous isotropic turbulence, where a simple dimensional argument yielded remarkable results, dimensional arguments were similarly used in this problem to obtain Eq. (1). A more careful analysis $[6,7]$ of the Navier-Stokes equation also supported the above result, which is one of the cornerstones in the theory of the turbulent boundary layer. Over the last two decades, much effort has been spent in providing a more microscopic justification for Kolmogorov's scaling in homogeneous isotropic turbulence, thereby enhancing our understanding of the energy cascade involved. Analysis of homogeneous isotropic turbulence has thus become a problem of nonequilibrium statistical physics [8-14]. In this work, we propose to use techniques from statistical physics to study the law of the wall and in the process provide calculations for the universal constant $\kappa$.

We begin by explaining the geometry and the traditional formulation. The flat plate (infinitely large) sits at $z=0$ (in the $x-y$ plane), and the flow (in the steady laminar situation) occurs in the $x$ direction, due to a parallel moving plate some distance away. We can view this as a two dimensional problem-the dynamics occurs only in the $x-z$ plane. Conti- nuity (for an incompressible flow) implies $(\partial u / \partial x)$ $+(\partial w / \partial z)=0$. We look for a situation which is $x$ independent, and hence $\partial w / \partial z=0$. The resulting constant value of $w$ is found to be zero since $w=0$ at $z=0$. In the laminar regime (low Reynold's number) the velocity $u$ satisfies $\nabla^{2} u=0$ and since the only dependence of $u$ is on $z$, we have $\partial^{2} u / \partial z^{2}$ $=0$ or a linear profile for $u$, i.e., $u=u_{0}(z / a)$, where $u_{0}$ and $a$ are constants. This is the usual Couette flow.

For high Reynold's number this flow becomes turbulent and the linear profile is restricted to a very small boundary layer. Somewhat away from the plate, the law of the wall shown in Eq. (1) is found. We explain the basis of our calcualtion in Sec. II. The De Dominicis-Martin model [9] for homogeneous, isotropic situation is introduced in Sec. III and a self-consistent calculation is described in Sec. IV. We generalize the model to the anisotropic situation in Sec. V, and obtain the constant in Sec. VI.

\section{BASIC HYDRODYNAMIC EQUATIONS}

In this section, we explain the basis of the calculation. Our starting point is the Navier-Stokes' equation for the velocity field $\vec{u}$,

$$
\frac{\partial \vec{u}}{\partial t}+(\vec{u} \cdot \vec{\nabla}) \vec{u}=-\frac{\vec{\nabla} P}{\rho}+\nu \nabla^{2} \vec{u},
$$

where $P$ is the pressure, which for an incompressible fluid is not an independent quantity. Using the incompressibility condition $\vec{\nabla} \cdot \vec{u}$, we see that $\nabla^{2} P / \rho=\vec{\nabla} \cdot[(\vec{u} \cdot \vec{\nabla}) \vec{u}]$, which locks the pressure to the velocity field. In the flow over a plate that we will consider, the plate will be taken to be in the $x-y$ plane with the mean velocity flowing in the $x$ direction under a maintained pressure gradient. It suffices to work with the two-dimensional geometry of the $x$-z plane, since all $y=$ const sections should appear identical. As is usual, we will split the velocity field into a mean and a fluctuating field, i.e.,

$$
\vec{u}=\vec{U}+\vec{v},
$$

with $\vec{v}$, the fluctuating part, having zero mean. Substituting Eq. (3) into Eq. (2), and taking averages, 


$$
\frac{\partial \vec{U}}{\partial t}+(\vec{U} \cdot \vec{\nabla}) \vec{U}+\langle(\vec{v} \cdot \vec{\nabla}) \vec{v}\rangle=-\frac{\vec{\nabla} P}{\rho}+\nu \nabla^{2} \vec{U}
$$

In this section we will use $x_{1}, x_{2}$, and $x_{3}$ as coordinates instead of $x, y$, and $z$, and the corresponding components of $\vec{U}$ and $\vec{v}$ will be $U_{1}, U_{2}$, and $U_{3}$ and $v_{1}, v_{2}$, and $v_{3}$, respectively. In the steady state, all average quantities are independent of $x_{1}$ and $x_{2}$, and, hence, from the incompressibility condition $\left(\partial U_{1} / \partial x_{1}\right)+\left(\partial U_{2} / \partial x_{2}\right)+\left(\partial U_{3} / \partial x_{3}\right)=0$, we infer that $U_{3}$ is independent of $x_{3}$ and since $U_{3}=0$ at $x_{3}=0$ (no-slip condition), it remains zero for all $x_{3}$. For the 1 component of Eq. (4), we have

$$
\begin{gathered}
U_{1} \frac{\partial U_{1}}{\partial x_{1}}+U_{3} \frac{\partial U_{1}}{\partial x_{3}}+\left\langle v_{1} \frac{\partial v_{1}}{\partial x_{1}}\right\rangle+\left\langle v_{2} \frac{\partial v_{1}}{\partial x_{2}}\right\rangle+\left\langle v_{3} \frac{\partial v_{1}}{\partial x_{3}}\right\rangle \\
=-\frac{\partial P}{\partial x_{1}} \frac{1}{\rho}+\nu \nabla^{2} U_{1},
\end{gathered}
$$

and from the 3 component of Eq. (4), we have

$$
\frac{\partial}{\partial x_{3}}\left(\frac{P_{0}}{\rho}+\left\langle v_{3}^{2}\right\rangle\right)=0
$$

where $P=P_{0}+p$. The above equation implies that $\left(P_{0} / \rho\right)$ $+\left\langle v_{3}^{2}\right\rangle$ is independent of $x_{3}$. From Eq. (5), we obtain

$$
\begin{aligned}
-\frac{\partial P_{0}}{\partial x_{1}} \frac{1}{\rho}+\nu \nabla^{2} U_{1}= & \left\langle v_{2} \frac{\partial}{\partial x_{2}} v_{1}\right\rangle+\left\langle v_{1} \frac{\partial}{\partial x_{1}} v_{1}\right\rangle \\
& +\left\langle v_{3} \frac{\partial}{\partial x_{3}} v_{1}\right\rangle+\left\langle v_{1} \frac{\partial}{\partial x_{3}} v_{3}\right\rangle \\
& -\left\langle v_{1} \frac{\partial}{\partial x_{3}} v_{3}\right\rangle .
\end{aligned}
$$

Now, considering $\left\langle v_{3}\left(\partial / \partial x_{3}\right) v_{1}\right\rangle+\left\langle v_{1}\left(\partial / \partial x_{3}\right) v_{1}\right\rangle$ $=\left(\partial / \partial x_{3}\right)\left\langle v_{1} v_{3}\right\rangle$ and $\left\langle v_{1}\left(\partial / \partial x_{3}\right) v_{3}\right\rangle=\left\langle v_{1}\left(\partial / \partial x_{1}\right) v_{2}\right\rangle$ $+\left\langle v_{1}\left(\partial / \partial x_{1}\right) v_{1}\right\rangle$, and using $\partial U_{1} / \partial x_{1}=U_{3}=\left(\partial / \partial X_{1}\right)\left\langle v_{1}^{2}\right\rangle$ $=0$, we have

$$
\left\langle v_{3} \frac{\partial}{\partial x_{3}} v_{1}\right\rangle=-\frac{\partial P}{\partial x_{1}} \frac{1}{\rho}+\nu \frac{\partial^{2} U_{1}}{\partial x_{3}^{2}}
$$

Now $\quad\left(\partial / \partial x_{3}\right)\left\langle v_{3} v_{1}\right\rangle=\left\langle v_{3}\left(\partial v_{1} / \partial x_{3}\right)\right\rangle+\left\langle\left(\partial v_{3} / \partial x_{1}\right) v_{1}\right\rangle$ $=\left\langle v_{3}\left(\partial v_{1} / \partial x_{3}\right)\right\rangle-\frac{1}{2}\left(\partial / \partial x_{1}\right)\left\langle v_{1}^{2}\right\rangle=\left\langle v_{3}\left(\partial v_{1} / \partial x_{3}\right)\right\rangle$, where we have used the equation of continuity for the fluctuating field, and thus, from Eq. (8),

$$
\nu \frac{\partial U_{1}}{\partial x_{3}}=\left\langle v_{1} v_{3}\right\rangle+\frac{1}{\rho} \frac{\partial P}{\partial x_{1}}+c .
$$

In writing the above equation we have used the fact that $P$ cannot depend on $x_{3}$-a fact which can be established by analysing the 3 component of Eq. (5). For a high Reynold's number flow, the stress tensor dominates, and we can drop the pressure term to write

$$
\nu \frac{\partial U_{1}}{\partial x_{3}}=\left\langle v_{1} v_{3}\right\rangle+c
$$

Far away from the wall, where the viscous stress is negligible, one can calculate the constant $c$, giving

$$
\nu \frac{\partial U_{1}}{\partial x_{3}}=\left\langle v_{1} v_{3}\right\rangle+\frac{1}{\rho} \frac{\partial P}{\partial x_{1}}+v_{0}^{2} .
$$

At this stage, following typical dimensional analysis, we proceed to establish the law of the wall. What the dimensional argument cannot obtain is the universal amplitude in the law of the wall. Our contribution is to actually calculate $\left\langle v_{1} v_{3}\right\rangle$ from the model of De Dominicis and Martin, and show that, for large $x_{3}$,

$$
\left\langle v_{1} v_{3}\right\rangle \simeq v_{0}^{2}\left[1-A \frac{x_{0}}{x_{3}}\right] .
$$

It should be noted that the above equation is valid only so long as $x_{3}$ lies within the boundary layer, which means that there is an upper limit as well as a lower limit to the value of $x_{3}$ for which Eq. (12) is valid. The important question for us is how to accomplish the calculation of $\left\langle v_{1} v_{3}\right\rangle$. The fluctuating velocity $v_{i}$ satisfies (with $\vec{\nabla} \cdot \vec{v}=0$ )

$$
\begin{aligned}
\dot{v}_{i}+\left(v_{j} \frac{\partial}{\partial x_{j}}\right) v_{i}= & -\frac{1}{\rho} \frac{\partial P}{\partial x_{i}}+\nu \nabla^{2} v_{i}+\left\langle v_{j} \frac{\partial}{\partial x_{j}} v_{i}\right\rangle \\
& -U_{j} \frac{\partial}{\partial x_{j}} v_{i}-v_{j} \frac{\partial}{\partial x_{j}} U_{i} .
\end{aligned}
$$

The last three terms on the right hand side make the calculation virtually impossible. To make analytic work possible under such circumstances, the randomly stirred model for fluctuating hydrodynamics was considered by De Dominicis and Martin [9] based on the work of Forster, Nelson, and Stephen [8]. In this model, the last three terms on the right hand side in Eq. (13) are replaced by a stochastic force $f_{i}$, whose correlation is specified in a manner such that the Kolmogorov spectrum [5] is obtained. For the homogeneous isotropic situation, we discuss the model in Sec. III.

\section{DE DOMINICIS-MARTIN MODEL}

The model for calculating correlation functions in a homogeneous isotropic situation is then given by

$$
\dot{v}_{i}+\left(v_{j} \frac{\partial}{\partial x_{j}}\right) v_{i}=-\frac{1}{\rho} \frac{\partial P}{\partial x_{i}}+\nu \nabla^{2} v_{i}+f_{i}
$$

To transfer the above equation into momentum space, we introduce the Fourier transforms, following notations as in Ref. [15],

$$
v(\vec{k}, \omega)=L t_{V, T \rightarrow \infty} \frac{1}{V^{1 / 2}} \frac{1}{T^{1 / 2}} \int_{V, T} v(\vec{x}, t) e^{i(\vec{k} \cdot \vec{x}-\omega t)} d^{D} x d t,
$$


and

$$
f(\vec{k}, \omega)=L t_{V, T \rightarrow \infty} \frac{1}{V^{1 / 2}} \frac{1}{T^{1 / 2}} \int_{V, T} f(\vec{x}, t) e^{i(\vec{k} \cdot \vec{x}-\omega t)} d^{D} x d t
$$

where $V$ and $T$ are the total volumes and times, respectively. Now, in momentum space, the equation reads

$$
\dot{v}_{i}(k)+\nu k^{2} v_{i}(k)=M_{i j l} v_{j}(p) v_{l}(k-p)+f_{1},
$$

where

$$
M_{i j l}(k)=\frac{i}{2 V^{1 / 2} T^{1 / 2}}\left[k_{j} P_{i l}(k)+k_{l} P_{i j}(k)\right],
$$

with the projection operator $P$ defined as

$$
P_{i j}(k)=\delta_{i j}-\frac{k_{i} k_{j}}{k^{2}}
$$

The correlation of the random force is specified as

$$
\begin{aligned}
\left\langle f_{i}\left(k_{1}, \omega_{1}\right) f_{j}\left(k_{2}, \omega_{2}\right)\right\rangle= & 2 D_{0} f\left(k_{1}\right) P_{i j}\left(k_{1}\right) \delta^{D}\left(\vec{k}_{1}+\vec{k}_{2}\right) \\
& \times \delta\left(\omega_{1}+\omega_{2}\right)
\end{aligned}
$$

where $D$ is the dimensionality of space, and $f\left(k_{1}\right)$ is a function which we need to specify to obtain agreement with the Kolmogorov spectrum.

Kolmogorov argued on the basis of dimensional analysis that in the inertial range, i.e., for length scales intermediate between the system size and the molecular size, the energy spectrum $E(k)$ is proportional to $k^{-5 / 3}$. If $\epsilon$ is the rate at which energy is supplied to the system, Kolmogorov's prediction was that

$$
E(k)=E_{0} \epsilon^{2 / 3} k^{-5 / 3},
$$

where $E_{0}$ is a dimensionless constant. If $E$ is the total energy per unit mass of the system, then

$$
\begin{aligned}
\int E(k) d k=E & =\frac{1}{2}\left\langle v^{2}(r)\right\rangle \\
& =\frac{1}{2 V T} \int d^{D} r d t v^{2}(r, t) \\
& =\frac{1}{2} \int \frac{d^{D} k}{(2 \pi)^{D}} \frac{d \omega}{2 \pi}\langle v(k, \omega) v(-k,-\omega)\rangle .
\end{aligned}
$$

This identifies

$$
E(k)=\frac{1}{2} \frac{S_{D}}{(2 \pi)^{D}} \int \frac{d \omega}{2 \pi}\langle v(k, \omega) v(-k,-\omega)\rangle k^{D-1},
$$

where $S_{D}$ is the surface area of a sphere in $D$ dimensions. From Eqs. (21) and (23), we conclude that

$$
\int \frac{d \omega}{2 \pi}\langle v(k, \omega) v(-k,-\omega)\rangle=\frac{C_{0} \epsilon^{2 / 3}}{k^{D+2 / 3}},
$$

where $C_{0}$ is a dimensionless constant. The dimensional analysis that led to Eq. (21), also allows us to obtain the form of the characteristic frequency scale of the fluctuations. If $\Gamma(k)$ is the relaxation rate, then dimensional analysis shows

$$
\Gamma(k)=\Gamma_{0} \epsilon^{1 / 3} k^{2 / 3}
$$

in the inertial region, with $\Gamma_{0}$ a dimensionless constant. Turning now to Eq. (17), the relaxation rate in the absence of the nonlinear term is easily seen to be $\omega(k)=\nu k^{2}$. In the presence of the nonlinear term, $\omega(k)$ is changed to the $\Gamma(k)$ term of Eq. (25) which implies that the constant molecular viscosity $\nu$ is changed to an effective viscosity $\nu_{e f f}(k)$ which can be written as $\nu_{e f f}(k)=\Gamma_{0} \epsilon^{1 / 3} k^{-4 / 3}$ in order to obtain agreement with Eq. (25). The role of the nonlinear term $\sum_{\vec{p}} M_{i j k} v_{j}(\vec{p}) v_{k}(\vec{k}-\vec{p})$ is to "dress" the nonlinear viscosity $\nu$ to the scale dependent viscosity $\nu_{e f f}(k)$ appropriate to the inertial range. An effective linearization of Eq. (17) then yields

$$
\dot{v}_{i}(k, t)+\nu_{e f f}(k) k^{2} v_{i}(k)=f_{i} .
$$

The correlation function is easily seen to be

$$
\begin{aligned}
\left\langle v_{i}(k, \omega) v_{j}\left(k^{\prime}, \omega^{\prime}\right)\right\rangle= & \frac{1}{-i \omega+\nu_{e f f} k^{2}} \frac{1}{-i \omega^{\prime}+\nu_{e f f} k^{\prime 2}} \\
& \times\left\langle f_{i}(k, \omega) f_{j}\left(k^{\prime}, \omega^{\prime}\right)\right\rangle \\
= & 2 D_{0} P_{i j}(k) f(k) \frac{1}{\omega^{2}+\nu_{e f f}^{2} k^{4}} \\
& \times \delta^{D}\left(\vec{k}+\vec{k}^{\prime}\right) \delta\left(\omega+\omega^{\prime}\right) .
\end{aligned}
$$

The integral of Eq. (24) is then given by

$$
\int \frac{d \omega}{2 \pi}\left\langle v_{i}(k, \omega) v_{i}(-k,-\omega)\right\rangle=\frac{D_{0} f(k)}{\nu_{e f f}(k) k^{2}}=\frac{D_{0} f(k)}{\Gamma_{0} \epsilon^{1 / 3} k^{2 / 3}},
$$

and, comparing with Eq. (20), we find

$$
f(k)=f_{0} \epsilon k^{-D} \text {. }
$$

This specifies the form of the correlation of the random force in Eq. (17) that would lead to the Kolmogorov spectrum.

Summarizing the above discussion on the randomly stirred model, we can say that the Kolmogorov consistent model is seen to be

$$
\dot{v}_{i}(k)+\nu k^{2} v_{i}(k)=\sum M_{i j l} v_{j}(p) v_{l}(k-p)+f_{i},
$$

with 


$$
\left\langle f_{i}(k, \omega) f_{j}\left(k^{\prime}, \omega^{\prime}\right)\right\rangle=\frac{2 F_{0} \epsilon}{k^{D}} P_{i j}(k) \delta^{D}\left(\vec{k}+\vec{k}^{\prime}\right) \delta\left(\omega+\omega^{\prime}\right),
$$

and an equivalent linearization of the equation of motion is

$$
\dot{v}_{i}(k)+\nu_{e f f} k^{2} v_{i}(k)=f_{i} .
$$

Analysis of the above model in the Kolmogorov range leads to the universal result

$$
\frac{\Gamma_{0}^{3}}{F_{0}}=\frac{3}{4} \frac{C_{D}}{(2 \pi)^{D}} \frac{D-1}{D+2},
$$

where $C_{D}$ is a constant again. In Sec. IV, we discuss various features of the randomly stirred model, and explain how the effective viscosity is obtained on the basis of a calculation starting from Eq. (17). These considerations are then used in Sec. V to extend the model to the situation where there is anisotropy due to the presence of the plate.

\section{SELF-CONSISTENT CALCULATION}

In this section, we undertake a self-consistent $[12,16]$ calculation starting from Eq. (17) to see how a $\nu_{\text {eff }}$ emerges automatically. We carry out diagrammatic perturbation expansions and apply self-consistency at one-loop level to obtain the nonlinear term induced relaxation rate $[16,17]$. The quantity of interest is the Green's function $G_{i j}(k, \omega)$, which is defined as

$$
\begin{aligned}
G_{i j}(k, \omega) & =P_{i j} G(k, \omega) \\
& =\left\langle\frac{\partial f_{i}(k, \omega)}{\partial v_{j}\left(k^{\prime}, \omega^{\prime}\right)}\right\rangle / \delta^{D}\left(k, k^{\prime}\right) \delta\left(\omega, \omega^{\prime}\right) .
\end{aligned}
$$

If we drop the nonlinear term (zeroth order), then the Green's function $G^{(0)}(k, \omega)$ is easily seen to be given by

$$
G^{(0)}(k, \omega)=\left(-i \omega+\nu k^{2}\right)^{-1} .
$$

The relation between the full Green's function and the bare function $G^{(0)}(k, \omega)$ is given by Dyson's equation

$$
G^{-1}=G_{0}^{-1}+\Sigma(k, \omega)=-i \omega+\nu k^{2}+\Sigma(k, \omega),
$$

where we have on purpose changed the sign in front of $\Sigma(k)$. In the above form, $\Sigma(k, \omega)$ is clearly the correction to the relaxation rate $\nu k^{2}$. If $\Sigma(k, 0)$ dominates $\nu k^{2}$, then the bare relaxation rate can be dropped, and $\Sigma(k)$ is the relaxation rate, with the Green's function given by $G^{-1}=-i \omega$ $+\Sigma(k, \omega)$. For the Kolmogorov case, discussed in Sec. III, we expect $\Sigma(k, 0)=\Gamma_{0} \epsilon^{1 / 3} k^{2 / 3}$, and this will dominate $\nu k^{2}$ as long as we consider wave numbers $k$ smaller than a critical $k_{D}$, given by

$$
\epsilon^{1 / 3} k_{D}^{2 / 3} \sim \nu k_{D}^{2},
$$

or

$$
k_{D} \sim \frac{\epsilon^{1 / 4}}{\nu^{3} / 4}
$$

The above $k_{D}$ is the wave number above which viscous dissipation becomes dominant. For wave numbers well below $k_{D}$ (true in the inertial range), $\Sigma(k, \omega)$ will dominate $\nu k^{2}$.

The first contribution $\Sigma^{(2)}(k, \omega)$ to $\Sigma(k, \omega)$ is obtained at the one-loop level, and is given by

$$
\begin{aligned}
\Sigma^{(2)}(k, \omega)= & \int \frac{d^{D} p}{(2 \pi)^{D}} \frac{d \omega^{\prime}}{2 \pi} M_{i j l}(k) G_{l m}^{(0)}\left(k-p, \omega-\omega^{\prime}\right) \\
& \times C_{j}^{(0)}\left(p, \omega^{\prime}\right) M_{i m n}(k) .
\end{aligned}
$$

At this point we convert this to a self consistent equation by making this an equation involving $\Sigma(k, \omega)$ alone. To do this we replace $\Sigma^{(2)}(k, \omega)$ by the full self-energy $\Sigma(k, \omega), G^{(0)}$ by the dressed Green's function $G(k, \omega)$ and $C^{(0)}$ by the dressed correlation function $C(k, \omega)$. This leads to

$$
\begin{aligned}
\Sigma(k, \omega)= & \frac{d^{D} p}{(2 \pi)^{D}} \frac{d \omega^{\prime}}{2 \pi} b(\vec{k}, \vec{p}, \vec{k}-\vec{p}) G\left(\vec{k}-\vec{p}, \omega-\omega^{\prime}\right) \\
& \times C\left(p, \omega^{\prime}\right),
\end{aligned}
$$

where $b(\vec{k}, \vec{p}, \vec{k}-\vec{p})$ is a factor which depends only on the angle between $\vec{k}$ and $\vec{p}$. $G$ on the right hand side is given by

$$
G^{-1}(k, \omega)=-i \omega+\Sigma(k, \omega),
$$

and the correlation function by

$$
C(k, \omega)=\frac{|G|^{2}}{k^{D}},
$$

which ensures that $\Sigma(k, \omega)$ is self-consistently determined from Eq. (39). We now ask whether $\Sigma(k, 0) \propto k^{2 / 3}$ is indeed a solution of Eq. (42). We write

$$
\begin{aligned}
\Sigma(k)= & k^{2} \int \frac{d^{D} p}{(2 \pi)^{D}} \frac{d \omega}{2 \pi} b(\vec{k}, \vec{p}, \vec{k}-\vec{p}) \\
& \times \frac{1}{p^{D}} \frac{1}{i \omega+\Sigma(\vec{k}-\vec{p}, \omega)} \frac{1}{\omega^{2}+\Sigma^{2}(p)} .
\end{aligned}
$$

Dimensional analysis of Eq. (39) shows that

$$
[\Sigma(k)]^{3} \sim k^{2}
$$

which is in accordance with Kolmogorov scaling.

The power-counting consistency of Eq. (42) satisfies the Kolmogorov spectrum, but does not automatically imply that this is the correct solution. We need to check whether the integral in Eq. (42) is finite or not. A potential problem of Eq. (42) is in the region of $p \simeq 0$. In this region of momentum space, if we perform, frequency integration, the domi- 
nant contribution to the integral will be from the small- $\omega$ part, where we may replace $i \omega+\Sigma(k-p, \omega)$ by $\Sigma(k)$, and then

$$
\Sigma^{2}(k) \simeq \frac{k^{2}}{(2 \pi)^{D}} \int \frac{d^{D} p}{p^{D}} \frac{1}{\Sigma(p)} b(k, 0, k) .
$$

The integral has to be cutoff at a lower limit $k_{0}$ for any $\Sigma(p)$ in order that it is finite, and we have

$$
\Sigma(k) \propto k .
$$

This dynamics is different from the Kolmogorov dynamics. It represents a sweeping process - a process where two eddies of very different scales interact, this interaction being simply a carrying of the smaller eddy by the larger one. The frequency scale associated with this process is simply $k$ [the time is proportional to the size of the eddy (Taylor's hypothesis)]. The Kolmogorov process, on the other hand, is concerned with interaction between neighboring eddies-the process that causes the energy to cascade from larger scales to shorter scales, and that gives a frequency scale proportional to $k^{2 / 3}$. In the problem of fluid flow over the infinite plate, one essentially has to consider eddies of all sizes in the two-dimensional plane, and hence the dynamics in the plane will be of the sweeping variety and should be governed by the frequency scale proportional to $k$, i.e., a characteristic frequency $[16,17]$ of $\nu_{e f f}(k) k^{2}$ with $\nu_{e f f}=\nu_{0} k^{-1}$.

We end this section with the observation that the cross correlation $\left\langle v_{1}(\vec{x}) v_{3}(\vec{x})\right\rangle$ that we are interested in vanishes in the homogeneous isotropic situation, since

$$
\begin{aligned}
\left\langle v_{1}(\vec{x}) v_{3}(\vec{x})\right\rangle & =\int \frac{d^{D} k}{(2 \pi)^{D}}\left\langle v_{1}(k) v_{3}(-k)\right\rangle \\
& =-\int \frac{d^{D} k}{(2 \pi)^{D}} \frac{k_{1} k_{3}}{k^{2}} \int \frac{d \omega}{2 \pi} C(k, \omega),
\end{aligned}
$$

and the last integral will vanish on angular integration. This is a consequence of the translational invariance that allows us to define the Fourier transform in all directions. In the presence of the plate, the translational invariance is broken in the $z$ direction and the above stress tensor is no longer zero.

\section{RANDOMLY STIRRED MODEL IN THE PRESENCE OF A BOUNDARY}

To set up a model corresponding to Eq. (21) for the situation where the flow is above a plate set on the $z=0$ plane, we note that we cannot work with the Fourier transform in the full three-dimensional space. Consequently the incompressibility condition would be difficult to implement. To circumvent this problem, we satisfy the incompressibility condition at the very outset by setting $\vec{v}=\vec{\nabla} \times \vec{A}$, and look for the equation of motion of the vector $\vec{A}$. We will work with the Fourier transform in the two-dimensional space, and expand $A_{i}(\vec{r}, t)$ as

$$
A_{i}(\vec{r}, t)=\sum A_{i}(\vec{k}, z, t) e^{i \vec{k} \cdot \vec{r}}
$$

where $\vec{k}$ and $\vec{r}$ are two-dimensional vectors in the $x-y$ plane. The first observation that one needs to make is that if $\vec{g}$ is the noise in the equation of motion for $\vec{A}$, then in the homogeneous isotropic situation the correlation of $\vec{g}$ would have to be prescribed as

$$
\left\langle g_{i}(k, \omega) g_{j}\left(k^{\prime}, \omega^{\prime}\right)\right\rangle=\frac{2 F_{0} \epsilon}{k^{D+2}} \delta_{i j} \delta^{D}\left(k+k^{\prime}\right) \delta\left(\omega+\omega^{\prime}\right),
$$

with $D=3$ in the $D$-dimensional space.

The boundary imposed at $z=0$ will cause anisotropy in the correlation of $g_{i}$ and $g_{j}$. We also note that $g_{i}(\vec{k}, \omega)$ will have to be replaced by $g_{i}(\vec{k}, z, \omega)$, where $\vec{k}$ is now a twodimensional vector, and we keep the coordinate space description in the $z$ variable. We thus write

$$
\begin{aligned}
\left\langle g_{i}\left(k, z_{1}, \omega\right) g_{j}\left(-k, z_{2},-\omega\right)\right\rangle & \\
= & \frac{2 F_{0} \epsilon}{k^{4}} G\left(z_{12}\right)\left[\delta_{i j}+\delta_{i 1} \delta_{j 3}+\delta_{i 2} \delta_{j 3}\right] \\
= & \frac{2 F_{0} \epsilon}{k^{4}}\left[\delta_{i j}+\delta_{i 1} \delta_{j 3}+\delta_{i 2} \delta_{j 3}\right],
\end{aligned}
$$

where $G\left(z_{12}\right)=$ const is the correct coordinate space description for obtaining the Kolmogorov spectrum. To write the equation of motion for $\vec{A}$, we use the equivalent linearization of Eq. (26), and write

$$
\frac{\partial A_{i}}{\partial t}+\nu_{e f f}\left[k^{2}-\frac{\partial^{2}}{\partial z^{2}}\right] A_{i}=g_{i}
$$

where the correlation of $g_{i}$ is prescribed in Eq. (49), and $\nu_{e f f}=\widetilde{\nu}_{0} k^{-1}$, as explained in the Sec. IV. The above equations are guaranteed to lead to the correct Kolmogorov description for the homogeneous isotropic situation in $D=3$. Our model is specified by Eqs. (50) and (49), and in Sec. VI will be used to calculate the correlation function $\left\langle v_{1}(\vec{x}) v_{3}(\vec{x})\right\rangle$.

\section{CORRELATION FUNCTION AND UNIVERSAL AMPLITUDE}

Our task in this section is to calculate the correlation function $\left\langle v_{1}(\vec{x}) v_{3}(\vec{x})\right\rangle$ on the basis of our model specified by Eqs. (50) and (49). Using $v_{i}=\epsilon_{i j k}\left(\partial / \partial x_{j}\right) A_{k}$, we have 


$$
\begin{aligned}
\left\langle v_{1}(\vec{r}, t) v_{3}(\vec{r}, t)\right\rangle= & \int \frac{d^{2} k}{(2 \pi)^{2}} \frac{d \omega}{2 \pi}\left\langle v_{1}(k, z, \omega) v_{3}(-k, z,-\omega)\right\rangle \\
= & \int \frac{d^{2} k}{(2 \pi)^{2}} \frac{d \omega}{2 \pi}\left\langle\left( i k_{2} A_{3}(k, z, \omega)\right.\right. \\
& \left.-\frac{\partial}{\partial z} A_{z}(k, z, \omega)\right)\left[-i K_{1} A_{2}(-k, z,-\omega)\right. \\
& \left.\left.+i k_{2} A_{1}(-k, z,-\omega)\right]\right\rangle \\
= & \int \frac{d^{2} k}{(2 \pi)^{2}} \frac{d \omega}{2 \pi} k_{2}^{2}\left\langle A_{3}(k z, z \omega)\right. \\
& \left.\times A_{1}(-k, z,-\omega)\right\rangle,
\end{aligned}
$$

since all the other correlations vanish due to the angular integrations. We can formally write the solution of Eq. (50) as

$$
A_{i}(k, z, \pm \omega)=\int \frac{G_{ \pm}\left(k, \pm \omega, z, z^{\prime}\right)}{\nu_{e f f}} F\left(z^{\prime}\right) d z^{\prime}
$$

and with this Eq. (51) becomes

$$
\begin{aligned}
\left\langle v_{1}(\vec{r}, t) v_{3}(\vec{r}, t)\right\rangle= & -\int \frac{d^{2} k}{(2 \pi)^{2}} \frac{d \omega}{2 \pi} k_{2}^{2} G_{+}\left(k, z, z_{1}, \omega\right) G_{-} \\
& \times\left(-k, z, z_{2},-\omega\right)\left\langle F_{3}\left(k, z_{1}, \omega\right) F_{1}\right. \\
& \left.\times\left(-k, z_{2},-\omega\right)\right\rangle d z_{1} d z_{2} \\
= & -2 F_{0} \epsilon \iint \frac{d^{2} k}{(2 \pi)^{2}} \frac{d \omega}{2 \pi} \frac{k_{2}^{2}}{k^{4}} \\
& \times \iint \frac{G_{+}\left(z, z_{1}\right) G_{-}\left(z, z_{2}\right)}{\nu_{e f f}^{2}} d z_{1} d z_{2} .
\end{aligned}
$$

The Green's function $G_{ \pm}$are obtained from the solution of

$$
\left(\frac{\partial^{2}}{\partial z^{2}}-k^{2} \mp i \Omega\right) G_{ \pm}=\delta\left(z-z^{\prime}\right)
$$

with $\Omega=\omega / \nu_{\text {eff }}$.

Defining $\kappa^{2}{ }_{ \pm}=k^{2} \pm i \Omega$, and working with boundary conditions appropriate to a single plate (i.e., $G$ vanishes on the plate and at infinity), we find [18]

$$
G_{ \pm}\left(z, z^{\prime}\right)=\frac{\sinh \left(\kappa_{ \pm} z_{<}\right) e^{-\kappa_{ \pm} z>}}{\kappa_{ \pm}} .
$$

Carrying out $z_{1}$ and $z_{2}$ integrations in Eq. (52), we obtain

$$
\begin{aligned}
\left\langle v_{1} v_{3}\right\rangle= & -2 F_{0} \epsilon \int \frac{d^{2} k}{(2 \pi)^{2}} \frac{d \omega}{2 \pi} \frac{k_{2}^{2}}{k^{4}} \frac{1}{\kappa_{+}^{2} \kappa_{-}^{2}} \frac{1}{v_{e f f}^{2}} \\
& \times\left(1-e^{-\kappa_{+} z}\right)\left(1-e^{-\kappa_{-} z}\right) .
\end{aligned}
$$

For large $\mathrm{z}$, the stress tensor tends to the constant value $-v_{0}^{2}$, which is given by

$$
\begin{aligned}
v_{0}^{2} & =F_{0} \epsilon \int \frac{d^{2} k}{(2 \pi)^{2}} \frac{d \omega}{2 \pi} \frac{\cos ^{2} \theta}{k^{2}} \frac{1}{\nu_{e f f}^{2}} \frac{1}{\kappa_{+}^{2} \kappa_{-}^{2}} \\
& =F_{0} \epsilon \int \frac{d^{2} k}{(2 \pi)^{2}} \frac{d \omega}{2 \pi} \frac{\cos ^{2} \theta}{\nu_{e f f}} \frac{1}{k^{4}} .
\end{aligned}
$$

In the sweeping time dominated situation $\nu_{\text {eff }}$ $=\Gamma_{0} \epsilon^{1 / 3} k^{-1} k_{0}{ }^{-1 / 3}$, and the above integral needs to be cutoff at a lower limit $k_{0}$, leading to

$$
v_{0}^{2}=\frac{F_{0}}{4 \pi} \frac{\epsilon^{2 / 3}}{k_{0}{ }^{2 / 3}} .
$$

It is this $v_{0}$ which sets the scale for the velocity at the boundary layer. We now need to specify the range of the $z$ space in which we are interested. As already discussed, the law of the wall in Eq. (1) holds somewhat away from the boundary. The relevant length scale is $k_{D}^{-1}=\nu\left(k_{0}\right) / v_{0}$ $=\sqrt{4 \pi} \Gamma_{0}^{3 / 2} k_{0}^{-1} / F_{0}^{1 / 2}$. Our interest is in the value of $z$ for which $k_{D} z>1$, and from Eq. (55) we find that the correction to $v_{0}^{2}$ is

$$
\left\langle v_{1} v_{3}\right\rangle=-v_{0}^{2}\left[1-\frac{5}{6} \frac{k_{D}}{k_{0}} \frac{1}{z_{+}}\right],
$$

where $z_{+}=k_{D} z$ (note that, for small $k_{0}, e^{-K_{0} z} \sim 1$ ). The quantity $F_{0} / \Gamma_{0}^{3}$ is a universal number known from the study of homogeneous isotropic turbulence, and given by Eq. (30), which, for $D=3$, yields

$$
\frac{\Gamma_{0}^{3}}{F_{0}}=\frac{3}{4} \frac{4 \pi}{8 \pi^{3}} \frac{2}{5}=\frac{3}{20 \pi^{2}} .
$$

Using this universal value, we have

$$
\left\langle v_{1} v_{3}\right\rangle=-v_{0}^{2}\left[1-\frac{5}{6} \sqrt{\frac{5 \pi}{3}} \frac{1}{z_{+}}\right] .
$$

Returning to Eq. (9), noting that $x_{3}$ there is our $z$ in the above discussion, we obtain

$$
\kappa=\frac{6}{5} \sqrt{\frac{3}{5 \pi}} \simeq 0.52 .
$$

The experimental values of $\kappa$ cluster around 0.42 .

The experimental data on the flow near a wall have accumulated since the time of Prandtl, and a comparatively recent computation was shown in a scaling plot by White [19]. In summary, we have extended the randomly stirred model of De Dominicis and Peliti to an anisotropic situation, to set up a scheme for calculating an universal amplitude appearing in 
a shear flow. The calculation is in the spirit of the Kolmogorov theory $[20,21]$, and corrections to Kolmogorov scaling [22] which form the main thrust in homogeneous isotropic turbulence are absent.

\section{ACKNOWLEDGMENTS}

One of the authors (A.K.C.) sincerely acknowledges partial financial support from C.S.I.R., India.
[1] L. Prandtl, Z. Angew. Math. Mech. 5, 136 (1925).

[2] L. Prandtl, Ergetn. Aerodyn. Versuchsanst 4, 18 (1932).

[3] T. von Karman, Proceedings of the Third International Congress on Applied Mechanics, Stockholm, 1930 (unpublished).

[4] P. Bradshaw, Turbulence, Topics in Applied Physics Vol. 12 (Springer-Verlag, New York, 1976).

[5] A.N. Kolmogorov, Dokl. Acad. Nauk SSSR 30, 301 (1941).

[6] J. O. Hinze, Turbulence (McGraw-Hill, New York, 1959).

[7] H. Tennekes and J. L. Lumley, A First Course In Turbulance (MIT Press, Cambridge, MA 1972).

[8] D. Forster, D. Nelson, and M. Stephen, Phys. Rev. A 16, 732 (1976).

[9] C. De Dominicis and P.C. Martin, Phys. Rev. A 19, 419 (1979)

[10] V. Yakhot and S.A. Orszag, Phys. Rev. Lett. 1, 1 (1986).

[11] D. Ronis, Phys. Rev. A 36, 3322 (1987).

[12] J.K. Bhattacharjee, J. Phys. A 27, L347 (1994); Phys. Fluids A
3, 879 (1991).

[13] E. Medina, T. Hwa, M. Kardar, and Y.C. Zhang, Phys. Rev. A 39, 3053 (1989).

[14] A.Kr. Chattopadhyay and J.K. Bhattacharjee, Europhys. Lett. 42, 119 (1998).

[15] H.W. Wyld, Ann. Phys. (N.Y.) 14, 143 (1961).

[16] C.Y. Mou and P. Weichman, Phys. Rev. Lett. 70, 1101 (1993).

[17] R.H. Kraichnan, J. Fluid Mech. 5, 497 (1959); 62, 305 (1974).

[18] J.K. Bhattacharjee, Phys. Rev. Lett. 77, 1524 (1996).

[19] F. M. White, Viscous Fluid Flow (McGraw-Hill, New York, 1991).

[20] U. Frisch, Turbulence: The Legacy of A. N. Kolmogorov (Cambridge University Press, Cambridge, 1995).

[21] V. Nikora, Phys. Rev. Lett. 83, 734 (1999).

[22] V.I. Belnicher, V.S. L’vov, and I. Procaccia, Physica A 254, 215 (1998). 